\title{
ARTHROSCOPIC MANAGEMENT OF PROXIMAL TIBIAL FRACTURES: TECHNICAL NOTE AND CASE SERIES PRESENTATION
}

\author{
HOREA BENEA, GHEORGHE TOMOAIA, ARTUR MARTIN, \\ CIPRIAN BARDAS
}

\author{
Department of Orthopedics and Traumatology, Iuliu Hatieganu University of \\ Medicine and Pharmacy, Cluj-Napoca, Romania
}

\begin{abstract}
Background and aims. The purpose of this article is to describe a new surgical method of arthroscopy assisted treatment of intraarticular proximal tibial fractures (ARIF - arthroscopic reduction and internal fixation), analyzing its efficiency and safety on a series of patients. Tibial plateau fractures affect the proximal tibial metaphyseal and articular surface, representing $1.2 \%$ of all fractures and up to $8 \%$ of all fractures in elderly.

Patients and method. Our case series consists of 6 patients with Schatzker types I-III tibial plateau fractures, treated in the Orthopedic and Traumatology Clinic of Cluj-Napoca from July 2012 to August 2014. Patients included in the study presented Schatzker type I-III tibial plateau fracture.

Results. The results obtained with the arthroscopic method were excellent in 5 cases (mean Rasmussen score 27.60 points) and good in 1 case (mean score 23.75). The radiological consolidation appeared after a mean of 12 weeks. No major complication was noted.

Conclusions. Diagnosis and treatment of associated lesions, shortening of hospitalization length and postoperative rehabilitation, but also the lower rate of complications, can make arthroscopic reduction and internal fixation the method of choice for the operative treatment of selected Schatzker I-III types of proximal tibial fractures.
\end{abstract}

Keywords: tibial fractures, treatment, arthroscopy, surgery.

\section{Background and aims}

The purpose of this article is to describe a new surgical method of arthroscopy assisted treatment of intraarticular proximal tibial fractures (ARIF - arthroscopic reduction and internal fixation), analyzing its efficiency and safety on a series of patients.

The proximal tibia is vulnerable because of its anatomical position and the complex injuries that occur at this level, affecting both bone and soft tissues (knee's articular cartilage, ligaments or menisci). Tibial plateau fractures affect the proximal tibial metaphysis and articular surface, representing $1.2 \%$ of all fractures and up to $8 \%$ in elderly [1].

Manuscript received: 03.03.2015

Accepted: 12.03.2015

Address for correspondence: beneahorea@yahoo.com
These fractures are produced as a result of the action of a deformation force in valgus/varus or axial compression. Pure splitting fractures are common especially in younger patients, in case of severe trauma, as the solid structure of subchondral bone is able to cope with the strong compressive force of the femoral condyle. With time, the dense cancellous bone in the tibial condyle is affected by osteopenia, it loses strength and will no longer resist to compressive forces. Splitting and depression fractures are more common in patients after the fifth decade, occurring in low energy trauma $[1,2]$.

The goal of the surgical treatment is to obtain a very good reduction of the fracture and a stable internal fixation, with low aggression and morbidity. The surgical approach should not create any further damage, especially of local blood supply, but it has to facilitate good visualization and 
adequate gestures in order to reposition the bony fragments and repair the associate soft tissue lesions. The implants must provide a stable construct, without being excessively bulky [3].

Treatmentoutcomescan be impaired by the restriction of articular motion, lack of articular congruence, stability or alignment restoration, and possible complications: vicious consolidation, algodystrophy, residual instability, infection, or osteoarthritis. These facts represent the main drawbacks of the classical surgical treatment by open reduction and internal fixation - ORIF.

\section{Patients and methods}

First we describe the surgical technique of ARIF: under spinal anesthesia and tourniquet, the patient is positioned supine on standard operating table and a leg holder is used above the knee to allow $0-120^{\circ}$ mobility. The arthroscopic tower and mobile $\mathrm{C}$-arm are placed before surgical field preparation. Standard arthroscopic AL/AM portals of the knee are used. Gravitational pressure lavage and arthroscopic shaver debridement are performed to evacuate the articular blood clots and small loose osteochondral fragments.

After this step, a full inspection of the joint will reveal associated lesions (menisci, articular cartilage, cruciate ligaments, etc) (Fig. 1). Then we continue with the evaluation of the fracture pattern, localization, extension in all three planes and degree of comminution (Fig. 2). The direct arthroscopic view shows a realistic comprehensive image of the MRI or CT-scan slices (Fig. 3 A,B).

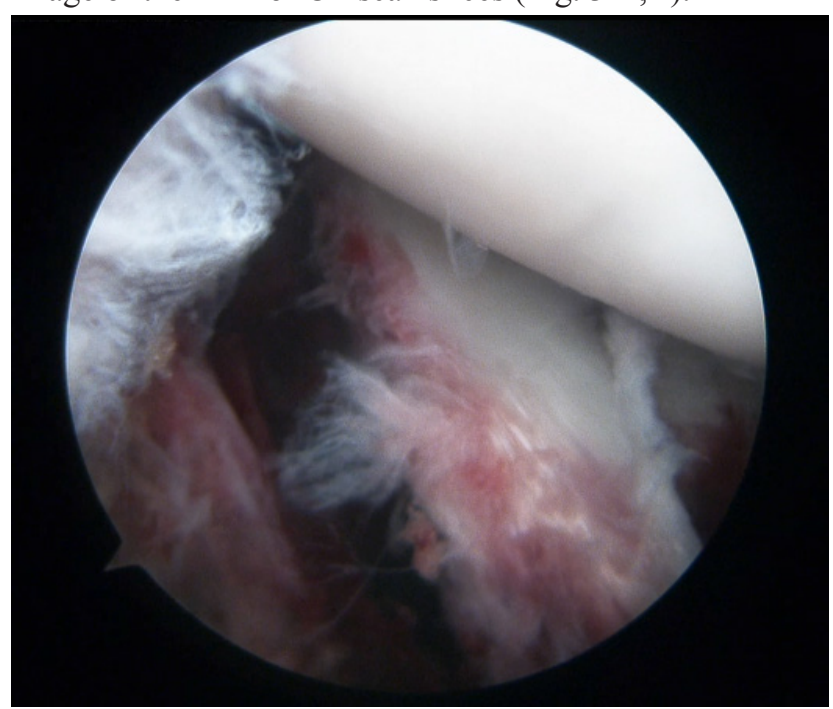

Figure 1. Fracture of the external tibial plateau with meniscal desinsertion - arthroscopic view.

Next we proceed to fracture reduction and internal fixation. The area of maximum depression of the tibial plateau must be identified and a $2 \mathrm{~mm} \mathrm{~K}$-wire is inserted from the metaphyseal region to the articular surface through the ACL ligamentoplasty tibial guide at sixty degrees of angle, and it will serve as a guide for medial corticotomy

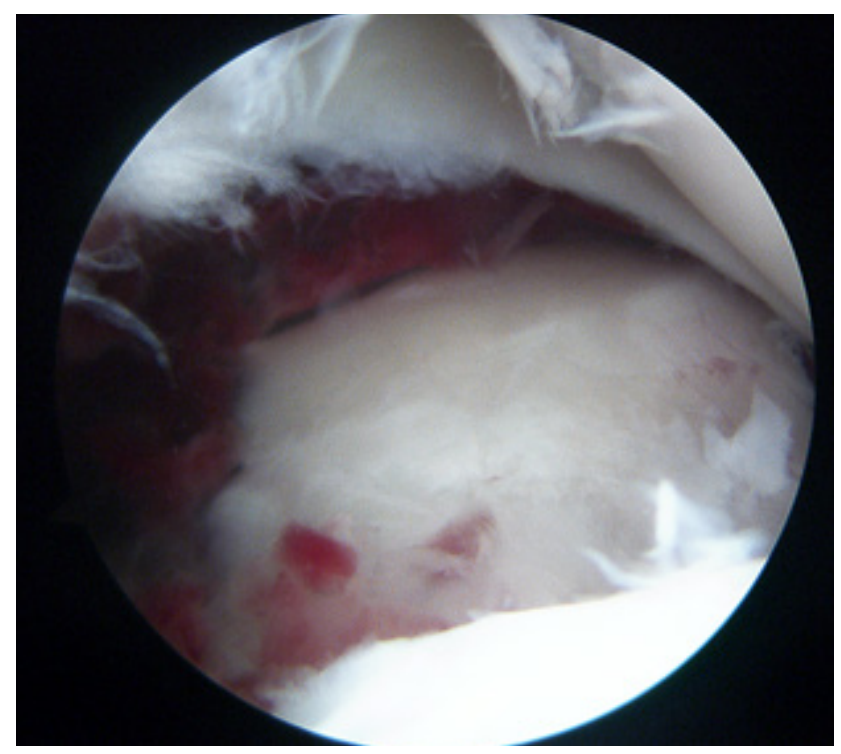

Figure 2. Depression type fracture of the external tibial plateau arthroscopic view.

of the tibia - performed using an 8-10 mm cannulated drill. With a cylindrical beater the depressed fragments are gently elevated altogether with the cancellous bone underneath, visualizing the restoration of the articular surface. The inferior facet of the meniscus serves as a level guide for articular surface height, which has to be also verified with the C-arm (Fig. 4).

The fixation is accomplished by two cannulated $6.5 \mathrm{~mm}$ cancellous screws, inserted in the proximity of the subchondral bone, to support the articular surface and prevent secondary displacement. Placement and progression of screws are controlled under image intensifier (Fig. 5).

In case of an important metaphyseal residual bone defect, it should be filled with 10-20 cc. of cancellous bone or bone graft substitutes. At the same time, the treatment of the associated meniscal lesion (suture or partial meniscectomy) or chondral lesion (debridement, microfractures) has to be performed.

Postoperatively we recommend immobilization of the knee in a fixed brace for the first 2-4 weeks, with alternative active mobilization $\left(0-90^{\circ}\right)$ protected by a mobile brace and muscular reinforcement, without weightbearing. Progressive partial weight bearing is allowed at about 8-12 weeks. Full weight-bearing is considered only after clinical and radiological consolidation of the fracture. Post-operative pain control and deep venous thrombosis prevention must also be done. The patients should be reviewed clinically and radiological at 4, 8, 12 weeks, 6 months, and 1 year after the intervention.

Our case series consists of 6 patients with Schatzker types I-III tibial plateau fractures, treated in the Orthopedic and Traumatology Clinic of Cluj-Napoca from July 2012 to August 2014. All of them signed the informed consent mandatory in our service and they were evaluated retrospectively. 

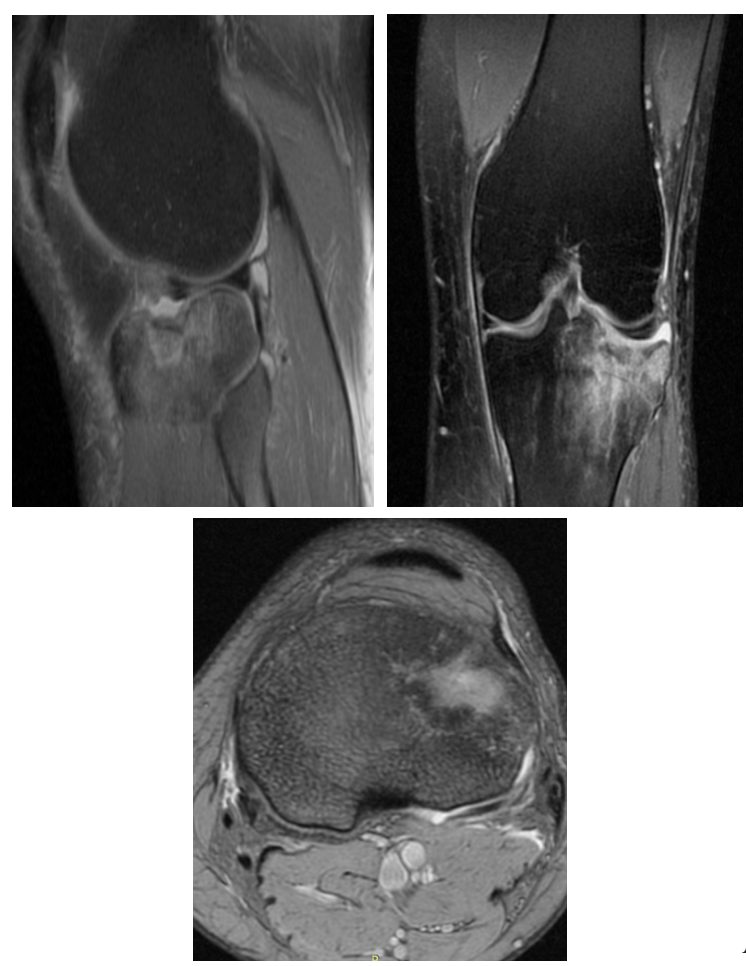

A.
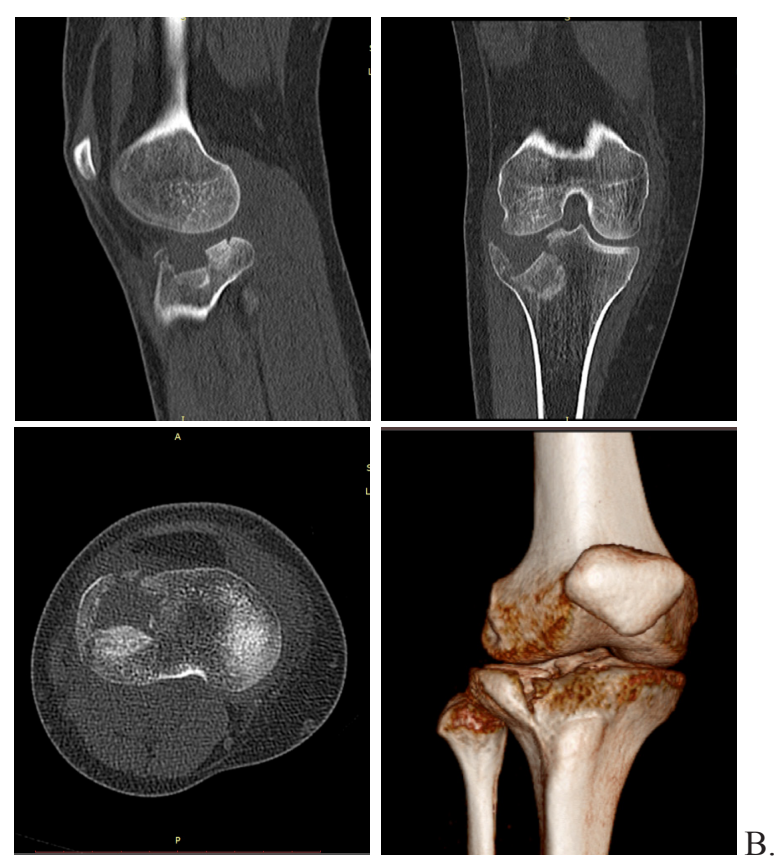

Figure 3. A. Anterolateral depression fracture of the external tibial plateau - MRI view.

B. Anterolateral profound depression fracture of the external tibial plateau - CT-scan view.

The patients mean age was 48 years and there were three men and three women. Two of them presented Schatzker I tibial plateau fracture, two patients Schatzker II fracture and the others two Schatzker III type. The diagnosis was established on standard radiographs of the knee (face and profile). For a better evaluation of the lesions we

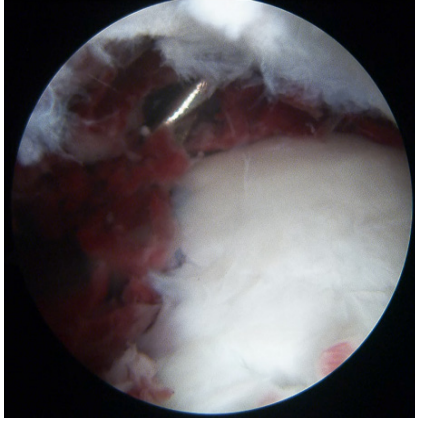

A.

Figure 4. Reduction of the fracture - arthroscopic view.

A. K-wire aiming of depression center.

B. Surface restoration by fragment elevation.
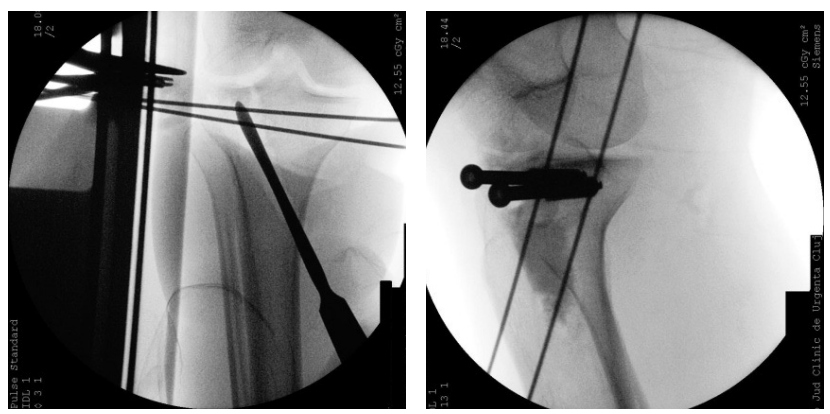

Figure 5. Intraoperative control of reduction and screws placement. performed preoperative CT-scan with 3D reconstruction in 4 cases, or preoperative MRI in the other 2. All the patients benefited from a fixation of the tibial plateau fracture under arthroscopic control according to the technique described above. They all followed the same postoperative rehabilitation protocol and they were evaluated at three months follow-up using the Rasmussen score.

\section{Results}

In five patients we practiced the internal fixation with 2 screws (Fig. 6A), but in one patient, due to the stability obtained by insertion of the bone substitute (Fig. $6 \mathrm{~B})$, we did not find necessary to perform osteosynthesis with screws.

The results obtained with the arthroscopic method were excellent in 5 cases (mean Rasmussen score 27.60 points) and just good in only 1 case (score 23.75). The radiological consolidation appeared after a mean of 12 weeks. We encountered a case of transitory edema of the calf, which lasted about three weeks, and a case of persistent articular effusion with flexion limitation. No case of compartment syndrome was registered.

Regarding the associated lesions, we found two external meniscal lesions, treated by suture in a case and minimal resection in the other. There were also three mirror chondral lesions of the lateral femoral condyle, for which we practiced arthroscopic debridement. We did not encounter any major complication intra or postoperatively. 

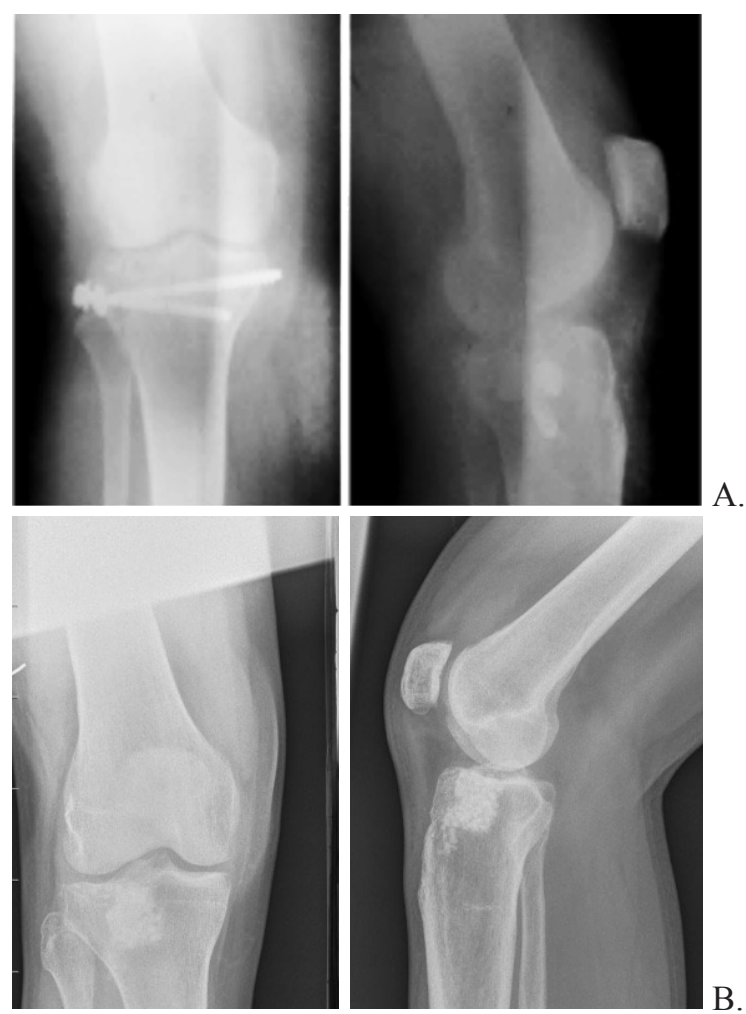

Figure 6 A. Reduction and osteosynthesis with two screws.

B. Reduction and filling with solid bone substitute.

\section{Discussion}

Recently emerged as a treatment option for tibial plateau fractures (Schatzker I-III), this method seems to be adopted by more and more surgeons in the world, as it benefits from the advantages of minimally invasive surgery - reduced hospitalization, faster recovery of knee mobility, reduced cutaneous complications $[2,4,5,6,7,8,9,10]$.

Regarding the fracture reduction, direct visualization of the articular surface is a major advantage because it allows an anatomical reduction without requiring an extensive arthrotomy. The lavage of the joint cavity is one of the most important steps of the procedure and should be done intensively. For the initial evaluation, a probe is used to elevate the lateral meniscus, as the fracture is localized frequently under the meniscus $[8,9,10]$.

Other lesions (external meniscus is damaged in about $30-50 \%$ of cases, lesions of cartilage or osteochondral lesions) can simultaneously benefit from specific treatment, improving patient recovery. Tears of the anterior cruciate ligament are also frequent, most of the time partial $[2,4,5,6,7,11]$.

Bone defect filling in case of younger patients is not mandatory. It is possible to use acrylic bone cement, autologous bone graft, allograft, or bone substitutes. Acrylic bone cement is very useful in aged patients, because it can associate filling of the cavity and immediate fixation of the bony fragment $[2,4,5,6]$.

Among the disadvantages of this method, we can include higher cost and duration of surgery and probably less rigid fixation than with plates. More thorough biomechanical studies are needed to better analyze this aspect, and analytical studies to compare the different methods of treatment and fixation of these fractures $[8,9]$.

\section{Conclusions}

The utilization of arthroscopy offers a good visualization of articular fracture reduction, allowing a stable fixation without the need of an extensive approach. Diagnosis and treatment of associated lesions (especially those of meniscus and articular cartilage), shortening of hospitalization duration and postoperative rehabilitation, but also the lower rate of complications, can make arthroscopy assisted reduction and internal fixation the method of choice for the operative treatment of selected Schatzker I-III types of articular proximal tibial fractures.

\section{Acknowledgement}

This paper was published under the frame of European Social Found, Human Resources Development Operational Program 2007-2013, project no. POSDRU/159/1.5/S/138776 - TRANSCENT.

\section{References}

1. Schatzker J, Tile M. The rationale of operative fracture care. 3rd edition, New York Springer Berlin, Heidelberg; 2005.

2. Siegler J, Galissier B, Marcheix PS, Charissoux JL, Mabit C, Arnaud JP. Percutaneous fixation of tibial plateau fractures under arthroscopy: a medium term perspective. Orthop Traumatol Surg Res. 2011;97(1):44-50.

3. Boisrenoult P, Bricteux S, Beaufils P, Hardy P. Screws versus screw-plate fixation of type 2 Schatzker fractures of the lateral tibial plateau. Cadaver biomechanical study. Rev Chir Orthop Reparatrice Appar Mot. 2000;86(7):707-711.

4. Duan XJ, Yang L Guo L, Chen GX, Dai G. Arthroscopically assisted treatment for Schatzker type I-V tibial plateau fractures. Chin J Traumatol. 2008;11(5):288-292.

5. Harris NL, Purnell ML, Pevny T, Larson AI. Arthroscopic management of tibial plateau fractures. Techniques in Knee Surgery. 2007; 6(1):9-16.

6. Kayali C, Oztürk H, Altay T, Reisoglu A, Agus H. Arthroscopically assisted percutaneous osteosynthesis of lateral tibial plateau fractures. Can J Surg. 2008;51(5):378-382.

7. Ruiz-Iban MA, Diaz-Heredia J, Elías-Martín E, Moros-Marco S, Cebreiro Martinez Del Val I. Repair of meniscal tears associated with tibial plateau fractures: a review of 15 cases. Am J Sports Med. 2012;40(10):2289-2295.

8. Lee HJ, Jung HJ, Chang EC, Lee JS, Jung HS. Second-look arthroscopy after surgical treatment of Schatzker type II plateau fractures through the lateral submeniscal approach. Arch Orthop Trauma Surg. 2014;134(4):495-499.

9. Chen L, Ma S, Li X. Minimally invasive treatment of tibial plateau fracture under arthroscopy monitoring. Zhongguo Yi Liao Qi Xie Za Zhi. 2014;38(3):232-234.

10. Fu F. Master Techniques in Orthopedic Surgery - Sports Medicine. Philadelphia: Wolters Kluwer; 2010:481-492.

11. Mustonen AO, Koivikko MP, Lindahl J, Koskinen SK. MRI of acute meniscal injury associated with tibial plateau fractures: prevalence, type, and location. AJR Am J Roentgenol. 2008;191(4):1002-1009. 